\title{
IAMJ
}

INTERNATIONAL AYURVEDIC MEDICAL JOURNAL

\section{ANTIHYPERTENSIVE EFFECT OF SOME MEDICINAL PLANTS IN AYURVEDA: A REVIEW}

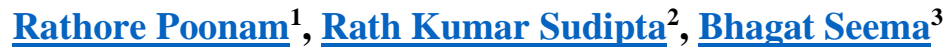 \\ ${ }^{1}$ MD Scholar, ${ }^{2}$ Asso. Professor, ${ }^{3}$ MD Scholar \\ Postgraduate Department of Dravyaguna Vigyana \\ National Institute of Ayurveda, Madav Vilas Palace, Amer Road, Jaipur (302002), Rajasthan, India \\ Corresponding Author: rathorepoonam345@gmail.com
}

\section{https://doi.org/10.46607/iamj2109072021}

(Published Online: July 2021)

Open Access

(C) International Ayurvedic Medical Journal, India 2021

Article Received: 22/06//2021 - Peer Reviewed: 24/06/2021 - Accepted for Publication: 26/06/2021

\section{Check for updates}

\begin{abstract}
The $21^{\text {st }}$ century is described as the age of anxiety and stress. This day-to-day stress and strain affect organs in the body through several psychophysical mechanisms. This is leading to the incidence of various psychosomatic diseases, such as the cardiovascular disorder like hypertension is quite significant. Hypertension is a multifactorial disease suffered by many people today. Current conventional treatments have done little to reduce the number of patients with hypertension because they are highly expensive and are usually associated with many side effects. About $80 \%$ of the world population relies on the use of traditional medicine, which is predominantly based on plant material. The present review aims to compile data and discuss numerous medicinal plants in Ayurveda possessing antihypertensive activity with other activities too.
\end{abstract}

Keywords: Hypertension, Medicinal Plants, Traditional Medicine, Antihypertensive Activity.

\section{INTRODUCTION}

Hypertension is a major risk factor for the development of cardiovascular disease and cerebrovascular disease, causing a high rate of mortality and morbidity. It mainly causes stroke and end-stage renal failure. It is asymptomatic but produces dreadful effects on the body. Hypertension is defined as a condition in which the blood vessels have persistently raised pressure beyond 140/90 $\mathrm{mm}$ of hg. The previously known high 
normal blood pressure is currently designated as Prehypertensive when the blood pressure is more than $120 / 80 \mathrm{~mm}$ of hg but less than 140/90 mm of hg. Blood pressure is the product of cardiac output and total peripheral vascular resistance. ${ }^{1}$ Hypertension is mainly classified as either primary (essential) and secondary. Primary hypertension is a condition with no medical causes and about 90 to $95 \%$ of cases are termed primary hypertension. The factors that lead to the development of this disease, vary considerably from patient to patient. Although no direct cause has identified itself, there are many factors such as sedentary lifestyle, stress, visceral obesity, potassium deficiency (hypokalemia), ${ }^{2}$ obesity, ${ }^{3}$ salts (sodium) sensitivity, ${ }^{4}$ alcohol intake, ${ }^{5}$ and vitamin D deficiency that increase the risk of high developing hypertension. ${ }^{6}$ The remaining 5 to $10 \%$ of cases, the cause of hypertension can be attributed to some underlying disease like Atherosclerosis, Acute nephritis etc. and is known as secondary hypertension. During 2015-2016, the prevalence of hypertension was $29.0 \%$ and the incidence increased with age viz. age group 18-39, 75\% and 40-59, 33.2\% and 60 above, $63.1 \% .^{7}$ The prevalence of hypertension in the urban Indian population was estimated to be $40.8 \%$ and that of hypertension in the rural population was $17.9 \% .{ }^{8}$ Globally the overall prevalence of hypertension in adults aged 25 and above was around $40 \%$ in 2008. ${ }^{9}$ Several physiological mechanisms are involved in the maintenance of normal blood pressure, and their derangement may play a part in the development of essential hypertension. A great many interrelated factors probably contribute to the raised blood pressure in hypertensive patients, and their relative roles may differ between individuals. Among the factors that have been intensively studied are salt intake, obesity and insulin resistance, the renin-angiotensin system, and the sympathetic nervous system. In the past few years, other factors have been evaluated, including genetics, endothelial dysfunction, low birth weight and intrauterine nutrition and neurovascular anomalies. ${ }^{10}$ Hypertension is mainly a lifestyle caused multifactorial disease. The following factors can play a role in the pathophysiology of hypertension.
1. Balance between Cardiac output and Peripheral Resistance.

2. Renin Angiotensin system.

3. Autonomic Nervous system.

4. Endothelial dysfunction.

5. Vasoactive substances like bradykinin, endothelin.

6. Genetic factors.

\section{Ayurvedic Perspective of Hypertension-}

Although no direct reference of any disease with the name having comparable signs and symptoms of hypertension is found in Ayurvedic classics, a review of previous theoretical and clinical works on this topic point to a certain mode of involvement of doshadushya in the genesis of it. Most of the efforts show a prime role of Vata in association with pitta and kapha. There are different opinions regarding the Ayurvedic nomenclature of this clinical condition such as Raktagatavata, Siragatavata, Avritavata etc. Acharya Charaka has advised treating such a disease without nomenclature by judging the involvement of doshadushya only. ${ }^{11}$

\section{Medicinal Plants and their necessity-}

Many plants that are introduced in the Ayurvedic system of medicine have been employing for the treatment of many ailments for thousands of years. According to World Health Organisation, herbal medicines are being used by $80 \%$ of the world population primarily in developing countries for primary health care. Herbal medicines are considered Harmless, no, more side effects and fewer adverse effects. It is estimated that about 7,500 plants are used in local health traditions. Out of these, the real medicinal value of the 4,000 plants is little known hitherto unknown to the mainstream population. The classical system of medicine such as Ayurveda, Siddha, Unani and Tibetian use about 1,200 Plants. Therefore, fewer side effects, better compatibility and only available treatment for some diseases make the herbal medicines an ideal remedy for the treatment of such diseases, ${ }^{12}$ and exploration of herbs that can be used for managing hypertension are of immense importance. 
Aim and Objectives- To compile and evaluate the Antihypertensive effect of medicinal plants in the Modern scientific data and with Ayurvedic properties.

\section{MATERIALS AND METHODS-}

The study comprises of:

a) Collection of literature in the Ancient Classical Texts, Scientific Journals, Research Papers,
PubMed, Reference books, World-wide accepted scientific databases, Dissertation etc. concerned with the concept.

b) The Antihypertensive Drugs, Hypertension disease, Traditional Medicinal Plants, words were used to search in the Online Databases.

\section{Effective Medicinal Plants on Hypertension-}

\begin{tabular}{|c|c|c|c|c|c|c|c|c|}
\hline $\begin{array}{l}\text { Sl. } \\
\text { No. }\end{array}$ & $\begin{array}{l}\text { Botanical } \\
\text { Name }\end{array}$ & Common Name & Family & Plant type & $\begin{array}{l}\text { Part } \\
\text { used }\end{array}$ & Chemical constituent & $\begin{array}{l}\text { Mechanism of } \\
\text { action }\end{array}$ & Formulation \\
\hline 1. & $\begin{array}{l}\text { Terminalia ar- } \\
\text { juna } \text { Roxb. }\end{array}$ & Arjuna & $\begin{array}{l}\text { Combre- } \\
\text { taceae }\end{array}$ & Tree & Bark & $\begin{array}{l}\text { Tannins, triterpenoid } \\
\text { saponins, flavonoids, } \\
\text { gallic acid, ellagic } \\
\text { acid, OPCs, phy- } \\
\text { tosterols, calcium, } \\
\text { magnesium, zinc and } \\
\text { copper. }\end{array}$ & $\begin{array}{l}\text { Vasorelaxation }{ }^{13} \\
\text { and dose-de- } \\
\text { pendent decrease } \\
\text { in arterial BP as } \\
\text { well as heart rate. }\end{array}$ & Bark extract \\
\hline 2. & $\begin{array}{l}\text { Convolvulus } \\
\text { pluricaulis } \\
\text { Chois. }\end{array}$ & Shankhapuspi & $\begin{array}{l}\text { Convol- } \\
\text { vulaceae }\end{array}$ & Shrub & $\begin{array}{l}\text { Whole } \\
\text { plant }\end{array}$ & $\begin{array}{l}\text { Convolvuline, con- } \\
\text { volidine, confoline, } \\
\text { volatile oils, hydro- } \\
\text { carbon, palmitic acid, } \\
\text { linoleic acid, D-glu- } \\
\text { cose, steroids-phy- } \\
\text { tosterols. }\end{array}$ & $\begin{array}{l}\text { Antidepressant } \\
\text { activity }^{14} \text {, anti- } \\
\text { stress activity }{ }^{15} \text {, } \\
\text { Cardioprotec- } \\
\text { tive, heart } \\
\text { strengthener and } \\
\text { control hyperten- } \\
\text { sion. }^{16}\end{array}$ & $\begin{array}{l}\text { Aqueous ex- } \\
\text { tract of the } \\
\text { plant }\end{array}$ \\
\hline 3. & $\begin{array}{l}\text { Nordostach- } \\
\text { yus jatamansi } \\
\text { DC. }\end{array}$ & Jatamansi & $\begin{array}{l}\text { Valeria- } \\
\text { naceae }\end{array}$ & Herb & Root & $\begin{array}{l}\text { Ursolic acid, nardosi- } \\
\text { nonediol, aristolen- } \\
\text { 9beta-ol, oleanolic } \\
\text { acid, beta-sitosterol. }\end{array}$ & $\begin{array}{l}\text { Jatamansi has } \\
\text { hypnotic and } \\
\text { CNS relaxant } \\
\text { properties. Jata- } \\
\text { mansi have many } \\
\text { properties hypo- } \\
\text { lipidemic, anti- } \\
\text { oxidant, seda- } \\
\text { tive, tranquilliz- } \\
\text { ing, antihyper- } \\
\text { tensive, antide- } \\
\text { pressant-like ac- } \\
\text { tivity, hypoten- } \\
\text { sive properties. }{ }^{17}\end{array}$ & $\begin{array}{l}\text { Aqueous ex- } \\
\text { tract of roots }\end{array}$ \\
\hline 4. & $\begin{array}{l}\text { Withania som- } \\
\text { nifera Dunal. }\end{array}$ & Ashwagandha & $\begin{array}{l}\text { Sola- } \\
\text { naceae }\end{array}$ & Shrub & Root & $\begin{array}{l}\text { Withanolides, hentri- } \\
\text { acontance, phy- } \\
\text { tosterol. }\end{array}$ & $\begin{array}{l}\text { Ashwagandha } \\
\text { contains chemi- } \\
\text { cals that might } \\
\text { help calm the } \\
\text { brain and reduce } \\
\text { lower blood pres- } \\
\text { sure. The Hypo- } \\
\text { tensive effect } \\
\text { mainly due to au- } \\
\text { tonomic gan- } \\
\text { glion blocking } \\
\text { action and that a } \\
\text { depressant action } \\
\text { on the higher cer- } \\
\text { ebral centres so } \\
\text { contributed to the } \\
\text { hypotension. }{ }^{18}\end{array}$ & Root extract \\
\hline 5. & $\begin{array}{l}\text { Boerhavia } \\
\text { duffusa Linn. }\end{array}$ & Punarnava & $\begin{array}{l}\text { Nyctagi- } \\
\text { naceae }\end{array}$ & Herb & Root & $\begin{array}{l}\text { Liridodendrin \& hy- } \\
\text { poxanthine. Punar- } \\
\text { navine }\end{array}$ & $\begin{array}{l}\text { Ca channel an- } \\
\text { tagonist }^{19}\end{array}$ & $\begin{array}{l}\text { Methanolic } \\
\text { extract of } \\
\text { roots }\end{array}$ \\
\hline 6. & $\begin{array}{l}\text { Tinospora } \\
\text { cordifolia } \\
\text { Willd. }\end{array}$ & Giloy & $\begin{array}{l}\text { Meni- } \\
\text { sperma- } \\
\text { ceae }\end{array}$ & $\begin{array}{l}\text { Climbing } \\
\text { shrub }\end{array}$ & $\begin{array}{l}\text { Stem, } \\
\text { root }\end{array}$ & $\begin{array}{l}\text { Alkaloids, phytoster- } \\
\text { ols, glycosides, tino- } \\
\text { sporaside, tinospora } \\
\text { acid, tinosporin. }\end{array}$ & $\begin{array}{l}\text { Tinospora cordi- } \\
\text { folia is known as } \\
\text { a medhya ra- } \\
\text { sayana (learning }\end{array}$ & Root extract \\
\hline
\end{tabular}




\begin{tabular}{|c|c|c|c|c|c|c|c|c|}
\hline & & & & & & & $\begin{array}{l}\text { and memory en- } \\
\text { hancer) in Ayur- } \\
\text { veda. The root of } \\
\text { T. cordifolia is } \\
\text { known to be used } \\
\text { traditionally for } \\
\text { its anti-stress ac- } \\
\text { tivity. }\end{array}$ & \\
\hline 7. & $\begin{array}{l}\text { Nigella sativa } \\
\text { Linn. }\end{array}$ & Kalongi & $\begin{array}{l}\text { Ranuncu- } \\
\text { laceae }\end{array}$ & Herb & Seed & $\begin{array}{l}\text { Thymoquinone, } \\
\text { dithymoquinone, } \\
\text { thymohydroquinone, } \\
\text { thymol, carvacrol, } \\
\text { tanethole and 4-ter- } \\
\text { pineol. }\end{array}$ & $\begin{array}{l}\text { Anti-atheroscle- } \\
\text { rosis, ACE inhib- } \\
\text { itor, nitrodila- } \\
\text { tors. }{ }^{21}\end{array}$ & $\begin{array}{l}\text { The oral dose } \\
\text { of an extract }\end{array}$ \\
\hline 8. & $\begin{array}{l}\text { Allium sa- } \\
\text { tivum Linn. }\end{array}$ & Lahsun & Liliaceae & $\begin{array}{l}\text { Bulb- } \\
\text { ous/Herb }\end{array}$ & $\begin{array}{l}\text { Flower } \\
\text { bud }\end{array}$ & $\begin{array}{l}\text { Minerals, enzymes, } \\
\text { amino acids, about } \\
33 \text { sulphur com- } \\
\text { pounds. }\end{array}$ & $\begin{array}{l}\text { Vasodilating ef- } \\
\text { fects on the ca- } \\
\text { rotid arteries and } \\
\text { aorta, reduces } \\
\text { blood pressure } \\
\text { and enhances the } \\
\text { diameter of ven- } \\
\text { ules and arteli- } \\
\text { oles }{ }^{22,23,24} \text { the in- } \\
\text { crease of nitric } \\
\text { oxide produc- } \\
\text { tion }{ }^{25} \text { ACE in- } \\
\text { hibitor }\end{array}$ & Dried garlic \\
\hline 9. & $\begin{array}{l}\text { Centella asiat- } \\
\text { ica (Linn.) Ur- } \\
\text { ban }\end{array}$ & Bharmmanduki & Apiaceae & Herb & $\begin{array}{l}\text { Whole } \\
\text { plant }\end{array}$ & $\begin{array}{l}\text { Saponins (Asiatic } \\
\text { acid, centelloside and } \\
\text { medecassosides), fla- } \\
\text { vonoid, amino acids, } \\
\text { tannins and sugar. }\end{array}$ & $\begin{array}{l}\text { The herbal ex- } \\
\text { tract reduces the } \\
\text { resting flux and } \\
\text { increases the } \\
\text { veno arterial re- } \\
\text { sponse. }^{26}\end{array}$ & Powder \\
\hline 10. & $\begin{array}{l}\text { Zingiber offic- } \\
\text { inalis Roscoe. }\end{array}$ & Adarak & $\begin{array}{l}\text { Zingiber- } \\
\text { aceae }\end{array}$ & Herb & $\begin{array}{l}\text { Rhi- } \\
\text { zome }\end{array}$ & $\begin{array}{l}\text { Volatile oil and se- } \\
\text { quiterpines (bbisabo- } \\
\text { lene zingiberene and } \\
\text { zingiberol) }\end{array}$ & $\begin{array}{l}\text { Induces } \mathrm{ca}^{+2} \\
\text { channel-block- } \\
\text { ing activity }\end{array}$ & Crude extract \\
\hline 11. & $\begin{array}{l}\text { Elaeocarpus } \\
\text { ganitrus Roxb. }\end{array}$ & Rudraksha & $\begin{array}{l}\text { Elaeocar- } \\
\text { paceae }\end{array}$ & Small tree & $\begin{array}{l}\text { Whole } \\
\text { plant }\end{array}$ & $\begin{array}{l}\text { Quercetin, rudrakin, } \\
\text { gallic acid, ellagic } \\
\text { acid }\end{array}$ & $\begin{array}{l}\text { Inhibits Angio- } \\
\text { tensin Convert- } \\
\text { ing Enzyme ac- } \\
\text { tivity }\end{array}$ & $\begin{array}{l}\text { Aqueous ex- } \\
\text { tract }\end{array}$ \\
\hline 12. & $\begin{array}{l}\text { Carum copti- } \\
\text { cum Benth. \& } \\
\text { Hook. }\end{array}$ & Ajwain & Apiaceae & Herb & Seeds & Thymol & $\begin{array}{l}\text { Produces a dose- } \\
\text { dependent fall in } \\
\text { arterial blood } \\
\text { pressure, fall in } \\
\text { BP and heart rate } \\
\text { (HR), and cal- } \\
\text { cium channel } \\
\text { blocking (CCB) } \\
\text { effect. }^{27}\end{array}$ & $\begin{array}{l}\text { Crude extract, } \\
\text { Juice orally. }{ }^{28}\end{array}$ \\
\hline 13. & $\begin{array}{l}\text { Cassia absus } \\
\text { Linn. }\end{array}$ & Chaksu & $\begin{array}{l}\text { Caesal- } \\
\text { piniaceae }\end{array}$ & Herb & $\begin{array}{l}\text { Leaves, } \\
\text { roots, } \\
\text { seeds }\end{array}$ & $\begin{array}{l}\text { Linoleic acid and lin- } \\
\text { olenic acids. }\end{array}$ & $\begin{array}{l}\text { Produces a dose- } \\
\text { related decrease } \\
\text { in systemic arte- } \\
\text { rial blood pres- } \\
\text { sure, accompa- } \\
\text { nied by a de- } \\
\text { crease in heart } \\
\text { rate. }\end{array}$ & $\begin{array}{l}\text { Methanolic } \\
\text { seed extract. }\end{array}$ \\
\hline 14. & $\begin{array}{l}\text { Cassia occi- } \\
\text { dentalis Linn. }\end{array}$ & Kashondi & $\begin{array}{l}\text { Caesal- } \\
\text { piniaceae }\end{array}$ & Small tree & Leaf & Alkaloids $^{30}$ & $\begin{array}{l}\text { Relaxation of } \\
\text { smooth muscle } \\
\text { and reduction of } \\
\mathrm{BP} \text { by inhibiting } \\
\mathrm{Ca}^{+2} \text { influx } \\
\text { through the re- } \\
\text { ceptor-operated } \\
\text { channel and volt- } \\
\text { age-sensitive } \\
\text { channel, show- } \\
\text { ing its non- }\end{array}$ & Leaf extract \\
\hline
\end{tabular}




\begin{tabular}{|c|c|c|c|c|c|c|c|c|}
\hline & & & & & & & $\begin{array}{l}\text { selectivity on } \\
\text { these } \mathrm{Ca}^{+2} \text { chan- } \\
\text { nels. }{ }^{31}\end{array}$ & \\
\hline 15. & $\begin{array}{l}\text { Daucus carota } \\
\text { Var. Sativa } \\
\text { DC. }\end{array}$ & Gajar & Apiaceae & Herb & $\begin{array}{l}\text { Aerial } \\
\text { part }\end{array}$ & $\begin{array}{l}\text { Two coumarin glyco- } \\
\text { sides coded as DC-2 } \\
\text { and DC- } 3 .{ }^{32}\end{array}$ & $\begin{array}{l}\text { Blockade of cal- } \\
\text { cium channels }\end{array}$ & $\begin{array}{l}\text { Intravenous } \\
\text { administra- } \\
\text { tion of these } \\
\text { glycosides }^{33} \text {. }\end{array}$ \\
\hline 16. & $\begin{array}{l}\text { Lavandula } \\
\text { stoechus Linn. }\end{array}$ & Uasthkhudus & $\begin{array}{l}\text { Aami- } \\
\text { aceae }\end{array}$ & Herb & $\begin{array}{l}\text { Flower } \\
\text { and oil }\end{array}$ & Essential oil $^{34}$ & $\begin{array}{l}\text { Calcium channel } \\
\text { blockers }\end{array}$ & Crude extract \\
\hline 17. & $\begin{array}{l}\text { Ocimum basil- } \\
\text { icum Linn. }\end{array}$ & Basil & $\begin{array}{l}\text { Lami- } \\
\text { aceae }\end{array}$ & Herb & $\begin{array}{l}\text { Leaves, } \\
\text { stem }\end{array}$ & $\begin{array}{l}\text { Linalol, eugenol, car- } \\
\text { yophyllene, rosma- } \\
\text { rinic, estragole and } \\
\text { methyl cinammate }\end{array}$ & $\begin{array}{l}\text { Blocking the cal- } \\
\text { cium channels }\end{array}$ & $\begin{array}{l}\text { Infusion } \\
\text { (crude ex- } \\
\text { tract) }\end{array}$ \\
\hline 18. & $\begin{array}{l}\text { Punica gran- } \\
\text { atum Linn. }\end{array}$ & Dadim & $\begin{array}{l}\text { Lyth- } \\
\text { raceae }\end{array}$ & Shrub & Fruit & $\begin{array}{l}\text { Polyphenols that in- } \\
\text { clude flavonoids, } \\
\text { condensed tannins } \\
\text { and hydrolyzable } \\
\text { tannins }{ }^{37}\end{array}$ & $\begin{array}{l}\text { Reduces the ac- } \\
\text { tivity of angio- } \\
\text { tensin-convert- } \\
\text { ing enzymes } \\
\text { (ACE) by about } \\
\text { and decreases } \\
\text { systolic blood } \\
\text { pressure. }\end{array}$ & Juice \\
\hline 19. & $\begin{array}{l}\text { Momordica } \\
\text { charantia } \\
\text { Linn. }\end{array}$ & Karela & $\begin{array}{l}\text { Curcurbi- } \\
\text { taceae }\end{array}$ & Herb & $\begin{array}{l}\text { Whole } \\
\text { plant }\end{array}$ & $\begin{array}{l}\text { Triterpenes, protein, } \\
\text { steroids, polyphe- } \\
\text { nols, alkaloids, lipids } \\
\text { and inorganics }\end{array}$ & $\begin{array}{l}\text { Reduce the sys- } \\
\text { temic BP and } \\
\text { heart rate }\end{array}$ & Maceration \\
\hline 20. & $\begin{array}{l}\text { Elettaria car- } \\
\text { damomum } \\
\text { Maton }\end{array}$ & Choti elaychii & $\begin{array}{l}\text { Zingiber- } \\
\text { aceae }\end{array}$ & Herb & $\begin{array}{l}\text { Seeds, } \\
\text { fruits }\end{array}$ & 1,8-cineole & $\begin{array}{ll}\text { Blocks } & \mathrm{Ca}^{+2} \\
\text { channels } & \end{array}$ & $\begin{array}{l}\text { Fruits pow- } \\
\text { der, crude ex- } \\
\text { tract }^{40}\end{array}$ \\
\hline 21. & $\begin{array}{l}\text { Tribulis ter- } \\
\text { restris Linn. }\end{array}$ & Gokharu & $\begin{array}{l}\text { Zygophyl- } \\
\text { laceae }\end{array}$ & Herb & Fruit & $\begin{array}{l}\text { Flavonoids, flavonol } \\
\text { glycosides, steroidal, } \\
\text { saponins and alka- } \\
\text { loids }\end{array}$ & $\begin{array}{l}\text { Increases } \mathrm{NO} \text {, } \\
\text { Reduces } \mathrm{ACE}^{41}\end{array}$ & $\begin{array}{l}\text { Aqueous ex- } \\
\text { tract }\end{array}$ \\
\hline 22. & $\begin{array}{l}\text { Viola odorata } \\
\text { Linn. }\end{array}$ & Banfasha & Violaceae & Hardy herb & Leaves & $\begin{array}{l}\text { Alkaloids, saponins, } \\
\text { tannins, phenolics, } \\
\text { coumarins and flavo- } \\
\text { noids }\end{array}$ & $\begin{array}{l}\text { Increases } \mathrm{NO} \\
\text { Regulates } \mathrm{Ca}^{+2}{ }^{42}\end{array}$ & Leaves extract \\
\hline 23. & $\begin{array}{l}\text { Pueraria lo- } \\
\text { bate Willd. }\end{array}$ & Kudzu & Fabaceae & Herb & Dry root & Isoflavones & $\begin{array}{l}\text { Attenuates car- } \\
\text { diac and aortic } \\
\text { hypertrophy, car- } \\
\text { diac fibrosis and } \\
\text { phospho- } \\
\text { ERK1/2 with a } \\
\text { mild reduction in } \\
\text { SBP. }\end{array}$ & $\begin{array}{l}\text { Ethanol ex- } \\
\text { tract of roots }\end{array}$ \\
\hline 24. & $\begin{array}{l}\text { Crocus sativus } \\
\text { Linn. }\end{array}$ & Saffron & Iridaceae & Herb & $\begin{array}{l}\text { Stigmas, } \\
\text { petals }\end{array}$ & $\begin{array}{l}\text { Flavonols, carote- } \\
\text { noids, phenolic com- } \\
\text { pounds, anthocya- } \\
\text { nins, terpenoids and } \\
\text { alkaloids, crocin, saf- } \\
\text { ranal, picrocrocin. }\end{array}$ & $\begin{array}{l}\text { Blocks } \quad \mathrm{Ca}^{+2} \\
\text { channels, re- } \\
\text { duces oxidative } \\
\text { stress. }\end{array}$ & $\begin{array}{l}\text { Aqueous and } \\
\text { ethanol ex- } \\
\text { tracts of Cro- } \\
\text { cus sativus } \\
\text { petals. }\end{array}$ \\
\hline 25. & $\begin{array}{l}\text { Andrographis } \\
\text { paniculata } \\
\text { Nees. }\end{array}$ & Kalmegh & $\begin{array}{l}\text { Acan- } \\
\text { thaceae }\end{array}$ & Herb & $\begin{array}{l}\text { Whole } \\
\text { plant }\end{array}$ & $\begin{array}{l}\text { 14-deoxy-11, } \\
\text { didehy- } \\
\text { drographolide, }\end{array}$ & $\begin{array}{lr}\text { Blocks } & \mathrm{Ca}^{+2} \\
\text { channels, } & \text { re- } \\
\text { duces ACE. } & \end{array}$ & $\begin{array}{l}\text { Extract of the } \\
\text { whole plant }\end{array}$ \\
\hline 26. & $\begin{array}{l}\text { Rauwolfia ser- } \\
\text { pentina Benth } \\
\text { ex. Kurz }\end{array}$ & Sarpagandha & $\begin{array}{l}\text { Apocyna- } \\
\text { ceae }\end{array}$ & Shrub & Root & $\begin{array}{l}\text { Rauwolfinine, re- } \\
\text { canescine, reserpine, } \\
\text { reserpine, serpentine, } \\
\text { ajmaline }\end{array}$ & $\begin{array}{l}\text { Adrenergic } \\
\text { blocking agent. } \\
\text { ACE inhibitor }\end{array}$ & Root extract \\
\hline
\end{tabular}

\section{DISCUSSION}

Hypertension is a serious disease affecting a significant population globally. Hypertension is managed in Ayurveda with herbs having vata-pitta shamaka action with an affinity to CNS and CVS. Ayurveda medicinal plants contain many phytochemicals that have been effective in lowering blood pressure and improving heart functions. The pharmacological activities of plants and their isolates affect the pathogenesis of hypertension by modulating several parameters like endothelial function, ROS production, pro-inflammatory signalling, platelet activation, opening and closing of ion channels, ACE inhibition. This review article documented several medicinal plants and their mode of 
action that have been reported to be effective in the management of hypertension in the field of Ayurveda. This study finds that the most researched and frequently utilized medicinal plants for the treatment of hypertension are Sarpagandha, Jatamamsi, Punarnava, Arjuna, Ashwagandha, Gokshura, kalmegh.

a) The chemical constituents of Andrographis paniculata 14-deoxy-11, 12-didehydroandrographolide decrease the level of $\mathrm{Ca}^{+2} .{ }^{46}$

b) The chemical constituents of Crocus sativus mainly cracin act as $\mathrm{Ca}^{+2}$ channel blockers and reduced heart rate. ${ }^{47}$

c) Antihypertensive activity of reserpine, Indole derivatives isolated from Rauwolfia serpentina can reduce both systolic and diastolic blood pressure. ${ }^{48}$

d) Punarnava contains active principles like Liridodendrin \& Hypoxanthine which are active antihypertensive agents, and the former is $\mathrm{Ca}^{+2}$ channel antagonist. It acts as a diuretic by increasing renal blood flow. ${ }^{49}$

Chemical constituents of above, mentioned herbs acts on CNS \& CVS, attenuates both systolic and diastolic blood pressure, ACE inhibitor and induces $\mathrm{ca}^{+2}$ channel-blocking activity. In other words, herbs having anti-hypertensive, stress-reducing, cardio-tonic, diuretic, antioxidant actions are used to manage this condition.

\section{CONCLUSION}

Natural medicinal products are considered in the case of primary healthcare because of better cultural acceptability, safety, potency, and lesser side effects. Several traditional herbal medicines and supplements have been recognized as potential therapeutic agents to manage hypertension and its associated complications. This review aims to document medicinal plants having potential Antihypertensive action given in Ayurveda. This compilation may help the Researchers, Pharmaceutical companies, and Investigators to further use these plants for Clinical research purposes.

\section{REFERENCES}

1. Chalmers J et al, WHO-ISH Hypertension Guidelines Committee. 1999 World Health Organization-
International Society of Hypertension Guidelines. J. Hypertens.1999, 17: 151- 183.

2. Kyrou I, Chrousos GP, Tsigos C. Stress, visceral obesity, and metabolic complications. Ann N. Y. Acad Sci, 2006, 1083: 77-110.

3. Wofford MR, Hall JE. Pathophysiology and treatment of obesity hypertension, Curr Pharma Design, 2004, 10: 3621-27.

4. Lackland DT, Egan BM, Dietary salt restriction and blood pressure in clinical trials. Curr Hypertens Rep, 2007, 9: 314-9.

5. Djousse L, Mukamal KJ, Alcohol consumption and risk of hypertension, Dose the type of beverage or drinking pattern matter. Rev Esp Cardiol, 2009, 62: 603-5.

6. Lee JH, O'Keefe JH, Bell D, Hensrud DD, Holick MF. Vitamin D deficiency an important, common, and easily treatable cardiovascular risk factor. J Am Coll Cardiol, 2008, 52: 1949-56.

7. Cheryl D. fryar, Yechian Ostchega, Craigm M. Hales and Deanna Kruszon-Moran, Prevalence of hypertension among adults, 2015-2016, available from: https:/www.cdc.gov.nchs/databriefs no. 289, October 2017.

8. Midhan Tanu et al, Prevalence of hypertension in India: A meta-analysis World J Meta-Anal, 2013, August 26, 1(2): 83-89.

9. World Health Organization, Global Health Repository available from: http:/www.who.int/gho/ncd/risk_factors/blood_pressure_prevalence_text/en/index.html.

Last accessed February 6, 2015.

10. Beevers G, Lip GY, O'Brien E. ABC of hypertension: The pathophysiology of hypertension. BMJ. 2001, 322 (7291): 912-916, DOI: 10. 1136/bmj.322.7291-912.

11. Pt. Kasinatha Pandaya and Dr Gorakha Natha Chaturvedi Ji, Agnivesh, Charaka Samhita with Vidyotini Hindi commentatory, Varanasi. Reprint year: 2013 Chaukhamba Bharati Academy, Sutra sthan chapter 18, Pg. 44-47.

12. Sohail, F. and M.N. Sohail. Despite its weedy nature is an important medicinal plant of NWEP, Pakistan. Int. J. Pharmacol, 2011. 7: 747-748.

13. Dwivedi S, Agarwal MP. Antianginal and cardioprotective effects of Terminalia arjuna, an indigenous drug in coronary heart disease. J Assoc Physi Ind. 1994, 42: 287-289.

14. International Research Journal of Pharmacy (ISSN 2230-8407), Review article of Velishala Hindu IRJP 2012.3(1). 
15. Dinesh Dhingra, Rekhavalecha, Evaluation of the antidepressant like activity of Convolvulus pluricaulis Chois. In the mouse forced swim and tail suspension tests (Med scimonit) 2007, 13(7): BR 155-161.

16. Singh 1997, Shukla 1981 a, 1981 b.

17. International Journal Med. Arom. Plants. ISSN 22494340 (Vol. 3, no. 1, PP 113-124, March 2013) [Rahman, H, Murlidharan, 2010] Comparative study of anti-depressant activity of methanolic extract of Nordostachys jatamansi DC. Rhizome on normal and sleep derived mice. De Pharmacia Lettre, 2(5): 441449.

18. Malhotra CL, Das PK, Dhalla NS, Prasad K. Studies on Withania ashwagandha, Kaul III. The effect of total alkaloids on the cardiovascular system and respiration. Indian J Med Res 1981, 49: 448-460.

19. Vandana S. Nade, Laxman A. Kawale, Anjali B. Tajanpure. Antihypertensive Potential of Boerhavia diffusa Linn. In Adrenaline-induced Hypertensive Model. International Journal of Experimental Pharmacology, 2015, 5(10): 24-30.

20. Reena Kulkarni et al, 2010 Apr-Jun, 1(2): 112-121, International Journal of Ayurvedic Research.

21. Prema Modak, Satyajit Halder, Bidduth Kumar Sarkar, Ananya Das, Arghya Prosun Sarkar and Sukalyan Kumar Kundu, Traditional Antihypertensive Medicinal Plants. World Journal of Pharmacy and Pharmaceutical Sciences, vol. 9, 884-912, 2020.

22. Lash, JP., LR. Cardoso, PM. Mesler, DA. Walczak and R. Pollak. The effect of garlic on hypercholesterolemia in renal transplant patients. Transplant. Proc, 1998, 30: 189-191.

23. Schulz, VR. Hansel and VE Tyler. Rational Phytotherapy, A Physicians Guide to Herbal Medicine. Springer, Berlin, Germany, 1979;306.

24. Korotkov, VM, The effect of garlic juice on blood pressure. Vrach. Delo, 1966; 6: 123.

25. 49 Plants That Treat High Blood Pressure. https:/wildfoodism.com/2014/01/49-plants-that-treat-highblood-pressure.

26. Prema Modak, Satyajit Halder, Bidduth Kumar Sarkar, Ananya Das, Arghya Prosun Sarkar and Sukalyan Kumar Kundu, Traditional Antihypertensive Medicinal Plants. World Journal of Pharmacy and Pharmaceutical Sciences, vol. 9, 884-912, 2020.

27. AH. Gilani, Q. Jabeen, MN. Ghayur, KH. Janbaz, MS. Akhtar. Studies on the antihypertensive, antispasmodic, bronchodilator and hepatoprotective activities of the Carum copticum seed extract, 2005, 8, 98(1-2): 127-35.

28. Mohammad Hossein Boskabady, Saeed Alitaneh and Azam Alavinezhad. Carum copticum, A Herbal Medicine with various Pharmacological effects. BioMed Research Internal, 2014, 11: ID 569087.

29. Saeed Ahmad, Ayesha Hassan, Waheed Mumtaz Abbasi, Tayyeba Rehman. Phytochemistery and pharmacological potential of Cassia absus- Areview. Journal of Pharmacy and Pharmacology, 2017, 70(1), 27-41.

30. Vijayalakshmi S, Ranjitha J, Devi Rajeswari V, Bhagiyalakshmi M. Pharmacological Profile of Cassia occidentalis Linn. - A Review. International Journal of Pharmacy and Pharmaceutical Sciences, 2013, 5(3): 29-33.

31. Ajagbonna OP, Majiminiyi FBO, Sofola OA. Relaxant effects of the aqueous leaf extract of Cassia occidentalis on rat aortic rings. Afr J Biomed Res, 2001, 4: 127-9.

32. Fu HW, Zhang L, Yi T, Feng YL, Tian JK. Two new guaiane-type sesquiterpenoids from the fruits of Daucus carota Linn. Fitoterapia, 2010, 81: 443-6.

33. Mahammad Shakheel B, Tripthi Saliyan, Satish S and Karunakar Hedge. Therapeutic Uses of Daucus carotaA Review. International Journal of Pharma and Chemical Research, 2017, 3(2): 138-143.

34. Lavender. $\quad$ www.rxlist.com/lavender/supplements.htm.

35. Azhar I, Aftab K, Usmanghani K. Naturally occurring calcium channel blockers. Hamdard Medicus, 1995, 38: 5-16.

36. Ibarrola DA, Montalbetti Y, Heinichen O, Alvarenga N, Figueredo A, Ferro EA. Isolation of hypotensive compounds from Solanum sisymbriifolium. J Ethnopharmacol, 70: 301-7.

37. Hamid Reza Rahimi, Mohammad Arastoo, and Seyed Nasser Ostad. A Comprehensive Review of Punica granatum Properties in Toxicological, Pharmacological, Cellular and Molecular Biology Research. Iran J Pharm Res, 2012, 11(2): 385-400.

38. Glauce SB. Viana, Silvana Magalhaes Siqueira Menezes, Luciana N. Cordeiro, F.J.A. Matos. Biological Effects of Pomegranate, especially its Antibacterial Actions Against Micro-organisms Present in the Dental Plaque and Other Infectious Processes. Bioactive Foods in Promoting Health, 2010, 457: 459-478.

39. Kumar DS, Sharathnath KV, Yogeswaran P, Harani A, Sudhakar K, Sudha P, Banji D. A medicinal potency 
of Momordica charantia. Int. J. Pharmaceut Sci Review Res, 2010, 1: 95-100.

40. Archana Sengupta and Shamee Bhattacharjee. Cardamom and its Active Constituent, I, 8-cineole. Molecular Targets and Therapeutic Uses of Spices, 2009, 6585.

41. Sharifi AM, Darabi R, Akbarloo N. Study of antihypertensive mechanism of Tribulus Terrestris in $2 \mathrm{~K} 1 \mathrm{C}$ hypertensive rats: role of tissue ACE activity. Life Sci., 2003, 73(23): 2963-71.

42. Hasan S Siddiqi, Malik H Mehmood, Najeeb U Rehman, and Anwar H Gilani. Studies on the antihypertensive and antidyslipidemic activities of Viola odorata leaves extract. Lipids Health Dis., 2012, 11:6.

43. Chunxiang Tan, Aimei Wang, Chan Liu, Yao Li, Yuepin Shi and Ming-Sheng Zhou. Puerarin Improves Vascular Insulin Resistance and Cardiovascular Remodeling in Salt- Sensitive Hypertension. The American Journal of Chinese Medicine, 2017, 45(6): 11691184.

44. H. A. El-Beshbishy, M.H. Hassan, M.A. Aly, S.A. Doghish, A.A. Alghaithy, Ecotoxicol. Environ. Saf. 83, 47-54 (2012). https://doi.org/10.10.16/j.ecoenv.2012.06.003

45. T. Jayakumar, C.-Y. Hsieh, J.-J Lee, J.-R. Sheu, Evid. Based Complement. Alternat. Med. 2013, 1-16 (2013). https://doi.org/10.1155/2013/846740

46. B. Salehi, A. Venditti, C. Frezza, A. Yucetepe, U. Altuntas, S. Uluata, M. Butnariu, I. Sarac, S. Shaheen, S.A. Petropoulos, K.R. Matthews, C.S. Kilic, M. Atanassova, C.O. Adetunji, A.O. Ademiluyi, B.Ozcelik, P.V.T. Fokou, N. Martins, W.C. Cho, J. Sharifi-Rad. Appl. Sci. 9, 3547-3586 (2019). https://doi.org/10.3390/app9173547

47. P.D.D. Dzeufiet, A. Mogueo, D.C. Bilanda, B-F.O. Aboubakar, L. Tedong, T. Dimo, P. Kamtchouing, MBC Complement. Altern. Med. 14, 507-519 (2019).

48. K. Kiran, A. J. Priya, R. G. Devi, Drug Invent. Today 10 (1), 2674-2678 (2018).

49. Kumar A, Singhal T, Upadhyay BN: PUNARNAVAA Potential Rejuvenator, Dav's Ayurveda, issue-20, vol. $\quad 2011$ w 2009 ww.davayurvedaezine.com/ezine/feb_2009/punarnava_a_poential_rejuvenator.php.

\section{ABBREVIATIONS}

BP- Blood pressure, SBP- Systolic blood pressure, $\mathrm{OPC}_{\mathrm{S}^{-}}$Oligomeric proanthocyanidins, ACE-
Angiotensin-converting enzyme, P-ERK- Phospho-intracellular signal-related kinase, CNS- Central nervous system, CVS- Cardiovascular system, ROS- Reactive oxygen species

\section{Source of Support: Nil Conflict of Interest: None Declared}

How to cite this URL: Rathore Poonam et al: Antihypertensive Effect Of Some Medicinal Plants In Ayurveda: A Review. International Ayurvedic Medical Journal \{online\} 2021 \{cited July 2021\} Available from: http://www.iamj.in/posts/images/upload/1458_1465.pdf 